\title{
Correction to: An Urban Crisis Management System for Critical Infrastructures: Participation Possibilities for Insurance Companies
}

\author{
Dirk Wrede $^{1}$ • Annemarie Will ${ }^{1}$ Tim Linderkamp ${ }^{2}$. \\ Johann-Matthias Graf von der Schulenburg ${ }^{1}$
}

Published online: 7 January 2019

(C) The Geneva Association 2019

\section{Correction to: The Geneva Papers, 2017, 42, (633-656) https://doi.org/10.1057/s41288-017-0069-9}

The authors wish to correct the numbering and formatting of the following passages in the printed article:

Page 637

Bush et al. and Leclaire and O'Reilly have designated the following essential functions of such a system to support decision-makers during the decision-making process:

(1) the cross-sectoral, functional representation of CI, including existing interdependencies (dependencies between single sectors or industries),

The original article can be found online at https://doi.org/10.1057/s41288-017-0069-9.

Dirk Wrede

dw@ivbl.uni-hannover.de

Annemarie Will

aw@ivbl.uni-hannover.de

Tim Linderkamp

tl@kvw-hannover.de

Johann-Matthias Graf von der Schulenburg

jms@ivbl.uni-hannover.de

1 Institute for Risk and Insurance, Gottfried Wilhelm Leibniz Universität Hannover,

Otto-Brenner-Straße 1, 30159 Hanover, Germany

2 Center for Risk and Insurance, Otto-Brenner-Straße 1, 30159 Hanover, Germany 
(2) the analysis of economic, safety-related and environmental consequences, as well as potential damage to human health, public security and national security from disaster or crises events, and

(3) the implementation of technically correct, appropriate, extensional and adaptable models and methods. ${ }^{36}$

Pages 637, 638

Four key challenges in the use of information systems or decision support systems in disaster and crisis management can be derived from these:

- The different data sets available in the event of a crisis are to be provided in a standardised data format to enable accurate and fast data access, analyses, evaluations and information dissemination.

- The information systems are designed to help decision-makers investigate complex and aggregated data, detect anomalies and extract analogies and correlations.

- The information systems should assist the responsible persons in risk detection and assessment during the crisis, as well as the selection of appropriate solution strategies.

- The information systems should provide different services to meet the requirements of the different emergency management phases and crisis events. ${ }^{37}$ 